\title{
Design and implementation of path planning for cleaning robot
}

\author{
Chen Chao-Da ${ }^{1, a^{*}}$, Chen Chui-Xin ${ }^{2, b}$, Chen Gong-Xing ${ }^{3, c}$ \\ ${ }^{1}$ Tianhe College of Guangdong Polytechnical Normal University, Guangzhou Guangdong China \\ ${ }^{1}$ Guangdong University of Technology, Guangzhou Guangdong China \\ ${ }^{2}$ Tianhe College of Guangdong Polytechnical Normal University, Guangzhou Guangdong China \\ ${ }^{3}$ Tianhe College of Guangdong Polytechnical Normal University, Guangzhou Guangdong China \\ agugu0769@126.com, b28477850@qq.com, ccandy448@126.com
}

Keywords: Robot; Infrared acquisition ; Path planning;

\begin{abstract}
The design of the main use of lightweight car to complete the cleaning robot path planning in reality, detection by infrared detection head, can be self completed indoor cleaning line. The robot through the infrared probe sensing the environment information, the establishment of the coverage map of the environment, and in the process of movement on the map update. On the other hand, in order to ensure the complete coverage of all areas at the same time, reduce the duplication of coverage, improve work efficiency, this paper puts forward square, saw form covering algorithm and spiral coverage algorithm, the three algorithms do not need complicated mathematical calculation, to ensure the real time of the algorithm, which reduces the memory requirements, at the same time, the algorithm does not make the entire search space and avoid the defects of the traditional grid algorithm as the space enlarges the real-time rapid decline.This topic is in this context, research on the path to cover more ground area is more important. Cleaning robot path planning there are many difficulties in application and design, such as low coverage, high repetition rate, so that the work efficiency is not high, so the subject has practical significance.
\end{abstract}

\section{Introduction}

In Europe and the United States and other developed countries, vacuum cleaner development earlier, wide range of applications, in recent years, has developed a variety of market oriented intelligent vacuum cleaner. Such as ICLEBO Germany volant Shi cleaning robot, Electrolux, Dr. Wei, the United States Rumba Roomba vacuum cleaner.

As a kind of service robot, cleaning robot can make people free from the heavy cleaning work, and has a wide application prospect. As satisfactory intelligent cleaning robot, it has automatically and thoroughly clean the home or office, it can take ground function, without the need of a bent operation; don't need people dragging wires are shuffled; people do not need to take it apart to accumulate in the interior of the garbage dumped out; don't need to be in next to endure the noise and the need of people just set by one way it works: one-time work still works every time, or the next day's work, the rest of the people would not have it. It can automatically charge, automatically put the internal garbage to a large volume of garbage can go. At the same time, it is still safe: there is no risk of electric shock, not damaged things, is not damaged, will not drop to under the stairs, don't go too far away and disappeared without a trace. Based on the above advantages, cleaning robot will be widely used in the home and work environment and public places, to achieve its high application value.

\section{2. system plan}

2.1 system structure design

The system design scheme is realized by using the model of the steering gear car model. In the electronic circuit design, it is the core of SCM, LM2596 and 6 infrared probe head as the information acquisition module and the two servo motor to form a cleaning robot model. 


\section{2 steering gear working principle}

Super load capacity, PWM control - the signal terminal as long as the input of a 50HZ signal, and then control the signal cycle of high pulse duration can control the car forward, backward, turn left, turn right, the 360 degree rotation, acceleration, deceleration and other functions. Greatly simplifies the design of intelligent car circuit.

Control method: $50 \mathrm{HZ}$ square wave signal a high level pulse duration corresponding to a speed. High level of $1 \mathrm{~ms} \sim 1.5$ milliseconds, the steering gear is rotating ( $1 \mathrm{~ms}$ is the fastest, the more close to $1.5 \mathrm{~ms}$ slower, 1.5 milliseconds when the steering gear to stop), high level for 1.5 milliseconds $~ 2$ milliseconds when the steering gear ( 1.5 seconds when the steering gear to stop, the more close to 2 MS reverse speed, $2 \mathrm{~ms}$ to the fastest speed inversion). Two servo with a potentiometer (open shell pry up the circuit board to see), can be high level pulse set at 1.5 milliseconds, observe whether the steering gear to stop, if you do not stop to adjust the potentiometer, until the stop, so as to complete the zero. The purpose of the zero adjustment: to keep the same pulse width of the same pulse in the same pulse.

Actuator output shaft and position feedback potentiometer is connected, steering wheel to rotate at the same time, driven by position feedback potentiometer, potentiometers output a voltage signal to the control circuit board, feedback, and control circuit board according to the degree of location determinants of motor rotation direction and speed, so as to achieve the goal to stop. The work flow of the control signal to control circuit board, the rotation of the motor, gear group deceleration, the steering wheel rotation, position feedback potentiometer to control circuit board feedback. The control signal of the servo system is a pulse width modulation (PWM) signal of 20MS, and the pulse width is from $0.5-2.5 \mathrm{MS}$ to 0 to 180 degrees. In other words, to provide a certain degree of pulse width, the output shaft will remain on a certain point of view, regardless of how the external torque is changed, until it provides a further width of the pulse signal, it will change the output angle to the new corresponding position. There is a reference circuit in the steering gear, the generation period is $20 \mathrm{MS}$, the width $1.5 \mathrm{MS}$ of the reference signal, there is a ratio of the signal and the reference signal is compared to determine the direction and size, so as to produce motor rotation signal. This shows that the servo is a position servo drive, rotation range can not exceed 180 degrees, for those who need to continue to change and can keep the driver, such as the robot's joints, aircraft and other aircraft.

2.3 infrared detection module

Infrared detection module directly affects the accuracy and quality of the robot work, so, what kind of external sensor selection is very important.

The sensor module to ambient light adaptation ability, which has a pair of infrared transmitting and receiving tubes, tube launched a certain frequency infrared. When detection direction meets the obstacle (reflector), infrared reflection back by the receiving tube receiving, after processing the comparator circuit, indicating the lantern lit, also signal output interface to output digital signal (a low level signal), through the potentiometer knob adjusts the detection distance, a range of effective distance $2 \sim 30 \mathrm{~cm}$, the working voltage of $3.3 \mathrm{v}-5 \mathrm{v}$. Detection range of the sensor can be through the potentiometer adjustment, with little interference, convenient assembly, convenient use etc., can be widely applied to robot obstacle avoidance, obstacle avoidance of car, line count and black and white line tracking and so on numerous occasions.

Sensor active infrared reflection detection, so the target reflectivity and shape is the key to the detection range. Black detection distance is the smallest, white is the largest; small area of small objects, large distance.

\section{3. path planning algorithm}

\section{1 robot path planning model}

Robot uses three kinds of path planning mode: box type, saw type, spiral movement path planning.Box type sa shown in figure1. 


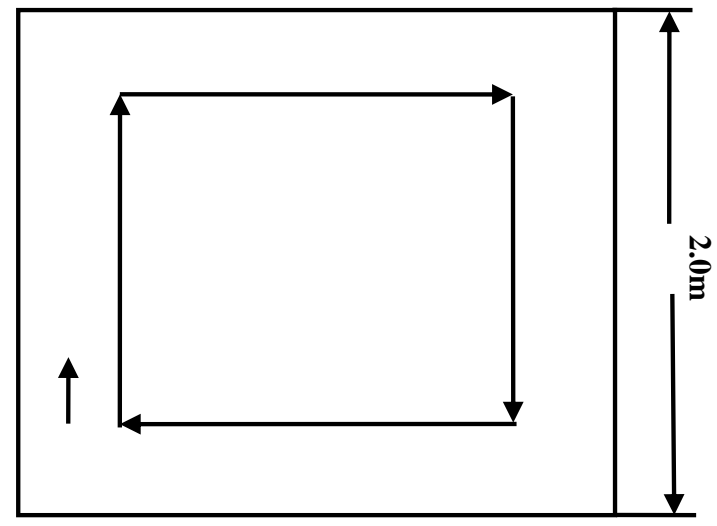

Figure 1 box type

Saw type as shown in figure 2.

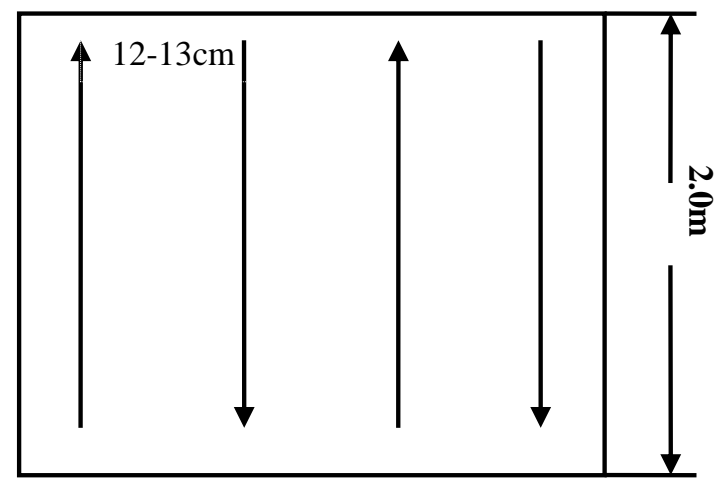

Figure 2 saw type

Internal spiral type as shown in figure 3.

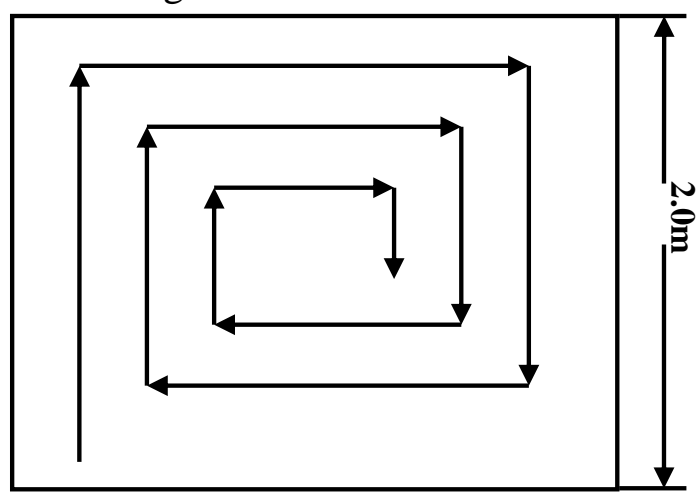

Figure 3 internal spiral type

3.2 by the photoelectric encoder installed on the robot angle calculation

A number of indexing for $\mathrm{N}$ encoder, the encoder receives the pulse number is $\mathrm{m}$, the diameter of the wheel is $\mathrm{D}$, a wheel turning angle $\theta$ :

$$
\theta=2 \pi m / \mathrm{N}
$$

Rolling wheels, on the edge of a point is equal to the distance around the wheel center distance and turned the wheel center motion:

$$
\mathrm{S}=\theta / 2=\pi \mathrm{md} / \mathrm{N}
$$

The position and orientation of the robot in the environment coordinate system $(\mathrm{X}(\mathrm{t}), \mathrm{Y}$ $(\mathrm{t}), \phi(\mathrm{t}))$ 。

Angle $\phi$ And right wheel rotation speed $\mathrm{v}_{\mathrm{R}}(\mathrm{t})$ 、Revolver speed $\mathrm{v}_{\mathrm{L}}(\mathrm{t})$, The distance between the two wheels has the following L:

$$
\mathrm{d} \phi / \mathrm{d} t=\left(\mathrm{v}_{\mathrm{R}}(\mathrm{t})-\mathrm{v}_{\mathrm{L}}(\mathrm{t})\right) / \mathrm{L}
$$


The corner values of the corner Zhi Hedi $\mathrm{n}+1$ times were the following relationship:

$$
\begin{gathered}
\phi_{n+1}-\phi_{n}=\frac{1}{L} \int_{n}^{n+T}\left(v_{R}(t)-v_{L}(t)\right) d t \\
=\frac{1}{L}\left(\Delta S_{R n}-\Delta S_{L n}\right) \\
\Delta \mathrm{S}_{\mathrm{Rn}}=\pi\left(\mathrm{m}_{\mathrm{Rn}+1}-\mathrm{m}_{\mathrm{Rn}}\right) \mathrm{d} / \mathrm{N} \\
\Delta \mathrm{S}_{\mathrm{Ln}}=\pi\left(\mathrm{m}_{\mathrm{Ln}+1}-\mathrm{m}_{\mathrm{Ln}}\right) \mathrm{d} / \mathrm{N}
\end{gathered}
$$

Of which: -- the right wheel distance; -- revolver distance difference. circle:

If the provisions of the robot when turning a wheel, a wheel forward, reverse, obstacle is the

$$
\begin{aligned}
\phi_{n+1}-\phi_{n} & =\frac{1}{L} \int_{n}^{n+T}\left(v_{R}(t)+v_{L}(t)\right) d t \\
& =\frac{1}{L}\left(\Delta S_{R n}+\Delta S_{L n}\right)
\end{aligned}
$$

Thus, by the formula (4) (7), the robot can be deduced from the angle of the robot's turn.

\section{Summary}

In this design, we study how to plan the clean path problem of the clean robot in the case of the environment map. Box, zigzag, and spiral cover method has been able to cover the room $98 \%$ of the area, a substantial increase in work efficiency. In the process of research, the application of each method is limited, so in the practical application, we should choose the most effective path planning method. However, with the rapid development of sensor technology and multimedia information fusion technology in recent years, it has greatly improved the positioning technology and environmental modeling technology. Once the environmental model has the characteristics of rapid establishment and rapid change, it is believed that the future path planning algorithm is more effective in the application of environmental map.

In this regard, the path planning technology has the following problems: 1 . In today's powerful hardware platform, the development of technology to establish a precise model of the environment, including some occasions need to build 3D environment model.

\section{References}

[1] Chen Zhaoda. Based on the serial communication technology of the manipulator motion control [J] manufacturing automation, 2013.

[2] Han Heng. The design of [J] intelligent cleaning robot AT89C51 based on the Gansu science and technology, 2008.

[3] Liang Xifeng. Path planning for indoor ground cleaning robot [J]. Journal of China Jiliang University, 2006.

[4] stone for people. Research on obstacle avoidance path planning for indoor cleaning robot [J]. computer application, 2007.

[5] Gong Jianwei. Heading tracking predictive control algorithm for wheeled mobile robot [J]. robot, 2001. 